This is the author's final version of the contribution published as:

Rocca, P; Galderisi, S.; Rossi, A.; Bertolino, A.; Rucci, P.; Gibertoni, D.; Montemagni, C.; Sigaudo, M.; Mucci, A.; Bucci, P.; Acciavatti, T.; Aguglia, E.; Amore, M.; Bellomo, A.; De Ronchi, D.; Dell'Osso, L.; Di Fabio, F.; Girardi, P.; Goracci, A.; Marchesi, C.; Monteleone, P.; Niolu, C.; Pinna, F.; Roncone, R.; Sacchetti, E.; Santonastaso, P.; Zeppegno, P.; Maj, M.. Social cognition in people with schizophrenia: A cluster-analytic approach.

PSYCHOLOGICAL MEDICINE. 46 (13) pp: 2717-2729.

DOI: $10.1017 /$ S0033291716001100

The publisher's version is available at:

http://www.journals.cambridge.org/abstract_S0033291716001100

When citing, please refer to the published version.

Link to this full text:

http://hdl.handle.net/2318/1613862 


\section{Title: Social cognition in people with schizophrenia: a cluster-analytic approach}

Paola Rocca1 ${ }^{1}$ Silvana Galderisi2, Alessandro Rossi ${ }^{3}$, Alessandro Bertolino4, Paola Rucci5, Dino Gibertoni5, Cristiana Montemagni1 ${ }^{1}$ Monica Sigaudo1, Armida Mucci², Paola Bucci2, Tiziano Acciavatti6, Eugenio Aguglia7, Mario Amore ${ }^{8}$, Antonello Bellomo9 , Diana De Ronchi10, Liliana Dell'Osso11, Fabio Di Fabio ${ }^{12}$, Paolo Girardi13, Arianna Goracci14, Carlo Marchesi ${ }^{15}$, Palmiero Monteleone ${ }^{16}$, Cinzia Niolu17, Federica Pinna ${ }^{18}$, Rita Roncone ${ }^{19}$, Emilio Sacchetti20, Paolo Santonastaso21, Patrizia Zeppegno22, Mario Maj2; Italian Network for Research on Psychoses* $^{*}$

\section{Affiliations}

1Department of Neuroscience, Section of Psychiatry, University of Turin, Turin, Italy;

2Department of Psychiatry, University of Naples SUN, Naples, Italy;

3Department of Biotechnological and Applied Clinical Sciences, Section of Psychiatry, University of L'Aquila, L'Aquila, Italy;

4Department of Neurological and Psychiatric Sciences, University of Bari, Bari, Italy;

5Department of Biomedical and Neuromotor Sciences, University of Bologna, Bologna, Italy;

6Department of Neuroscience and Imaging, Chair of Psychiatry, G. d'Annunzio University, Chieti, Italy;

7Department of Clinical and Molecular Biomedicine, Psychiatry Unit, University of Catania, Catania, Italy;

${ }^{8}$ Department of Neurosciences, Rehabilitation, Ophthalmology, Genetics and Maternal and Child Health, Section of Psychiatry, University of Genoa, Genoa, Italy;

9Department of Medical Sciences, Psychiatry Unit, University of Foggia, Foggia, Italy;

10Department of Biomedical and Neuromotor Sciences, Section of Psychiatry, University of Bologna, Bologna, Italy;

11Department of Clinical and Experimental Medicine, Section of Psychiatry, University of Pisa, Pisa, Italy;

12Department of Neurology and Psychiatry, Sapienza University of Rome, Rome, Italy;

${ }^{13}$ Department of Neurosciences, Mental Health and Sensory Organs, S. Andrea Hospital, Sapienza University of Rome, Rome, Italy;

${ }^{14}$ Department of Molecular Medicine and Clinical Department of Mental Health, University of Siena, Siena, Italy;

15Department of Neuroscience, Psychiatry Unit, University of Parma, Parma, Italy;

16Department of Medicine and Surgery, Chair of Psychiatry, University of Salerno, Salerno, Italy;

17Department of Systems Medicine, Chair of Psychiatry, Tor Vergata University of Rome, Rome, Italy;

${ }^{18}$ Department of Public Health, Clinical and Molecular Medicine, Section of Psychiatry, University of Cagliari, Cagliari, Italy; 
${ }^{19}$ Department of Life, Health and Environmental Sciences, Unit of Psychiatry, University of L'Aquila, L'Aquila, Italy;

20Psychiatric Unit, School of Medicine, University of Brescia, and Department of Mental Health, Spedali Civili Hospital, Brescia, Italy;

21Psychiatric Clinic, Department of Neurosciences, University of Padua, Padua, Italy;

22Department of Translational Medicine, Psychiatric Unit, University of Eastern Piedmont, Novara, Italy

*The members of the Italian Network for Research on Psychoses are listed in the Appendix

\section{Corresponding Author}

Paola Rocca, M.D.

Department of Neuroscience, Unit of Psychiatry

University of Turin

Via Cherasco 11, 10126 Turin, Italy

Tel: 0039-011-6634848

Fax: 0039-011-673473

e-mail: paola.rocca@unito.it 


\section{Abstract}

Background: The study aimed to subtype patients with schizophrenia on the basis of social cognition (SC), and to identify cut-offs that best discriminate among subtypes in 809 outpatients recruited in the context of the Italian Network for Research on Psychoses (NIRP).

Methods: A two-step cluster analysis of The Awareness of Social Inference Test (TASIT), the Facial Emotion Identification Test (FEIT) and Mayer-Salovey-Caruso Emotional Intelligence Test (MSCEIT) scores was performed. Classification and regression tree (CRT) analysis was used to identify the cut-offs of variables that best discriminated among clusters.

Results: We identified three clusters, characterized by unimpaired (42\%), impaired $(50.4 \%)$, and very impaired (7.5\%) SC. Three Theory of Mind (TOM) domains were more important for the cluster definition as compared to emotion perception and emotional intelligence. Patients more able to understand simple sarcasm ( $\geq 14$ for TASIT-SS) were very likely to belong to the unimpaired SC cluster. Compared to patients in the impaired SC cluster, those in the very impaired SC cluster performed significantly worse in lie scenes (TASITLlk10), but not in simple sarcasm. Moreover, functioning, neurocognition, disorganization and SC had a linear relationship across the three clusters, while positive symptoms were significantly lower in patients with unimpaired SC as compared to patients with impaired and very impaired SC. On the other hand, negative symptoms were highest in patients with impaired levels of SC.

Conclusions: If replicated, the identification of such subtypes in clinical practice may help in tailoring rehabilitation efforts to the person's strengths to gain more benefit to the person. 


\section{Introduction}

Social cognition (SC) refers broadly to the domains of cognitive functions that are employed in socially relevant situations (Harvey and Penn, 2010). These include emotion processing, social perception, theory of mind/mental state attribution, and attributional style/bias, as well as more complex and developing concepts such as social metacognition (Pinkham et al., 2015).

Schizophrenia patients demonstrate significant deficits across multiple dimensions of SC (Savla et al., 2013). Growing evidence indicates that SC impairment is large in effect size, appears to be present in the prodrome (or high risk samples), in first-degree relatives of patients with schizophrenia, during early course, and during periods of symptom remission (Bora et al., 2013; de Acha'val et al., 2010; Kohler et al., 2010; Fett et al., 2011; Green et al., 2011), suggesting that SC deficits in schizophrenia are likely core features of the illness and not simply a result of medication side-effects or clinical episodes (Ventura et al., 2013).

These disturbances have been found to be largely independent of positive symptoms but may be more strongly related to disorganized and negative symptoms (Fett and Maat 2013; Ventura et al., 2013). Though neurocognitive (NC) and SC tasks share some cognitive processes and therefore are often correlated, NC and SC have been observed to exist as distinct constructs (Sergi et al., 2007; van Hooren et al.,2008; Mehta et al., 2013).

Numerous studies corroborated that both SC and NC are related to everyday functioning (Fett et al., 2011). SC impairments in schizophrenia have recently been explored as potential mediators of the relationship between NC and functioning (Brekke et al., 2005; Horton and Silverstein, 2008; Schmidt et al., 2011; Galderisi et al., 2014), and as a more proximal target for psychosocial rehabilitation. Recent findings from a metaanalysis indicate that SC has a stronger relationship with functional outcome than NC (Fett et al., 2011).

It appears that considerable heterogeneity exists in the area of SC. Thus, it is possible that subtypes with different levels and patterns of SC performance may exist, but little research has been conducted to identify these subtypes (Nelson et al., 2007; Bell et al., 2013). One method that may be useful in identifying subtypes is cluster analysis. Cluster analysis provides an opportunity to group individuals using a data-driven approach rather than pre-determined grouping criteria (e.g. diagnosis). Such approaches permit individuals to be classified based not on single variables or factors but on patterns or profiles of traits, creating the potential for more homogeneous groupings than single domains or predefined categories. Cluster-analytic studies on NC in patients with schizophrenia have identified different clusters with differing levels of NC dysfunction, i.e., one neuropsychologically normal cluster, one severely and broadly impaired cluster, one to three intermediate profiles of mixed NC deficits (Barton et al., 2009). Few analytic studies on SC have been conducted in schizophrenia (Nelson et al., 2007; Bell et al., 2013). The first one used cluster analysis to determine if subtypes with different levels and patterns of performance are present across a variety of social and general perception tasks in one hundred inpatients with schizophrenia (Nelson et al., 2007). Two homogenous 
subtypes were identified, that showed performance deficits across measures of emotion, face, and general perception compared with normative data, supporting the presence of a generalized deficit model as regards social and general perception tasks in schizophrenia. Cluster 1 included the most severely impaired patients, who showed scores roughly three SD below that of normal controls on visual perception. Moreover, they showed higher levels of thought disorder than cluster 2; cluster 2 showed mild to moderate levels of impairment with scores about one SD below normal.

The second study, carried out in a sample of 77 outpatients with schizophrenia (Bell et al., 2013), identified three clusters: one with high negative symptoms (HN), one with low negative symptom and higher SC (HSC), and one with low negative symptoms and poorer SC (LSC). The HSC cluster had a significantly higher proportion of patients ever being married compared with the other two clusters, and the LSC cluster had a higher proportion of married than the HN clusters. The LSC cluster contained more patients with more than two arrests. Moreover, the HSC cluster had an earlier reported age of onset and significantly more hospitalizations. GAF scores were significantly better for the HSC cluster compared with the other 2 groups. Lifetime substance abuse also differed significantly among clusters, with the LSC cluster having the highest proportion and the HN group the lowest.

The goals of this study are to analyze the pattern of SC variables in schizophrenia using cluster analysis, to examine the relationship of real-life functioning, demographic characteristics, and psychopathology with cluster membership, and to identify cut-offs that best discriminate among clusters.

Several limitations of previous studies were addressed in the present investigation. We investigated a large, and well-characterized sample of patients with schizophrenia recruited in the context of a multicenter study of the Italian Network for Research on Psychoses (NIRP). A full assessment of different aspects of SC was carried out, including emotional intelligence, emotion recognition and theory of mind (TOM).The MATRICS (Measurement and Treatment Research to Improve Cognition in Schizophrenia) Consensus Cognitive Battery (MCCB) was chosen for NC assessment, as it is regarded as the "state of the art" neuropsychological battery for research purposes in schizophrenia (Kern et al., 2008; Nuechterlein et al., 2008, 2013a, 2013 b).

Measurement of everyday functioning was accomplished through two general approaches: ratings of real-life functioning using the Specific Levels of Functioning Scale (SLOF) (Schneider and Struening, 1983; Mucci et al, 2014), and objective measures of functioning, including the achievement of specific milestones (stable relationship, employment, and residential status) (Harvey et al., 2012, 2013). The SLOF was endorsed by the panel of experts involved in the Validation of Everyday Real-Life Outcomes (VALERO) initiative as a suitable measure of real-life functioning (Harvey et al., 2011; Leifker et al., 2011).

\section{Materials and Methods}

\section{Participants}


Eight-hundred and nine study participants were recruited from patients with a diagnosis of schizophrenia according to DSM-IV, living in the community and consecutively seen at the outpatient units of 26 Italian university psychiatric clinics and/or mental health departments (Galderisi et al. 2014). Inclusion and exclusion criteria have been described in a previous paper (Galderisi et al., 2014). The control group included 780 healthy subjects matched with patients by gender, age range and geographical area of origin and recruited through flyers from the community at the same sites as the patient sample. Inclusion criteria were the absence of a current or lifetime Axis-I or II diagnosis and exclusion criteria were a) a history of head trauma with loss of consciousness; b) a history of moderate to severe mental retardation or of neurological diseases; c) a history of alcohol and/or substance abuse in the last six months; d) current pregnancy or lactation; e) inability to provide an informed consent.

All participants signed a written informed consent to participate after receiving a detailed explanation of the study procedures and goals.

\section{Measures}

\section{Psychopathology}

The Positive and Negative Syndrome Scale (PANSS) was used to rate symptom severity. Scores for the dimensions "disorganization" and "positive symptoms" were calculated based on the consensus 5-factor solution proposed by Wallwork et al. (2012).

Negative symptoms were assessed using the Brief Negative Symptom Scale (BNSS). The Italian version of the scale was validated as part of the NIRP project (Mucci et al., 2015). In line with previous research (Kirkpatrick et al. 2011; Strauss et al., 2012) domains evaluated by the scale loaded on two factors: "avolition" (A), consisting of anhedonia, asociality and avolition, and "poor emotional expression" (DE), including blunted affect and alogia.

\section{Social cognition}

The assessment of SC included a test contained in the MCCB: the Mayer-Salovey-Caruso Emotional Intelligence Test (MSCEIT) (Mayer et al., 2002), managing emotion section, which examines the regulation of emotions in oneself and in one's relationships with others by presenting vignettes of various situations, along with ways to cope with the emotions depicted in these vignettes. It was integrated by the Facial Emotion Identification Test (FEIT) (Kerr et al., 1993), which examines emotion perception, and The Awareness of Social Inference Test (TASIT) (McDonald et al., 2006), which is a TOM test consisting of 7 scales (positive emotions, negative emotions, sincere, simple sarcasm, paradoxical sarcasm, sarcasm enriched, lie), organized into three sections: Emotion recognition; Social Inference (minimal); Social Inference (enriched). The manual of the TASIT was translated into Italian by a psychiatrist of the Department of Psychiatry of the University of Naples SUN who gained experience in the use of the English version of the instrument during his 
stage at the Department of Psychiatry and Biobehavioral Sciences at University of California, Los Angeles (UCLA), as part of his PhD Course. The videotaped vignettes of the TASIT were dubbed in Italian at the Fono Roma Studios (www.fonoroma.com), a prestigious society in the field of film industry. As to the FEIT, the adaptation of the Italian version required the translations of the six emotions reported on the screen above the stimuli.

\section{Neurocognition}

The MCCB includes tests for the assessment of six distinct cognitive domains: processing speed, attention/vigilance, working memory, verbal learning, visual learning, reasoning and problem solving.

\section{Real-life functioning}

The SLOF includes the following domains: physical efficiency, skills in self-care, interpersonal relationships, social acceptability, community activities, and working skills. The Italian version of the scale has recently been validated (Mucci et al., 2014) in the context of the NIRP.

\section{Definition of milestones}

Functional milestones were defined in line with Harvey et al. (2012) and included social outcomes such as ever being married, living with spouse/partner, currently or previously engaged, which we categorized as current or former relationship vs. none.

Vocational outcome was categorized as employed vs. not employed and residential outcome was defined as living without supervision.

\section{Statistical analysis}

A two-step cluster analysis of the seven TASIT scale scores and the FEIT and MSCEIT scores was performed to identify patient subgroups with different SC profiles. The two-step algorithm has several desirable features that differentiate it from the traditional hierarchical cluster analysis or k-means cluster analysis: first its ability to analyze large datasets efficiently, second the selection of the number of clusters based on a goodness of fit index, third its ability to use categorical and continuous variables. The two-step algorithm has several desirable features that differentiate it from the traditional hierarchical cluster analysis or k-means cluster analysis: first its ability to analyze large datasets efficiently, second the selection of the number of clusters based on a goodness of fit index, third its ability to use categorical and continuous variables. Given the large sample size, we chose to use this technique rather than hierarchical cluster analysis. In two-step cluster analysis, cases are first assigned to 'preclusters' and then preclusters are used for a second-step hierarchical analysis. Variables were transformed to z-scores using the Italian normative sample data. The normative sample was recruited from each geographic macro-area (Northern, Central and Southern Italy) and was stratified by age, gender, and education in order to have a demographic composition similar to that described in the last published census by the Italian National Census Bureau ${ }^{37}$. Two-Step Cluster Analysis procedure uses a likelihood 
distance measure which assumes that variables in the cluster model are independent. Further, each continuous variable is assumed to have a normal (Gaussian) distribution. However, this procedure is fairly robust to violations of both the independence and the distributional assumptions (Norušis, 2005). In addition, the Euclidean distance can be used, which is suitable when variables are continuous.

In the first step, the procedure builds a Cluster Features (CF) Tree. The tree begins by placing the first case at the root of the tree in a leaf node that contains variable information about that case. Each successive case is then added to an existing node or forms a new node, based upon its similarity to existing nodes and using the distance measure as the similarity criterion. In the second step, the leaf nodes of the CF tree are then grouped using an agglomerative clustering algorithm. The agglomerative clustering can be used to produce a range of solutions. To determine which number of clusters was "best", each of these cluster solutions was compared using Schwarz's Bayesian Criterion (BIC) as the clustering criterion.

Functioning, psychopathology, NC scale scores and demographic characteristics were compared among clusters using ANOVA, followed by Tamhane post-hoc tests, to allow for heterogeneity of variance, and categorical variables were compared among clusters using $X^{2}$ test. Bonferroni correction was applied to the significance level to adjust for multiple comparisons. The correlation between milestones was examined using $\varphi$ correlation coefficient and the correlation between milestones and clusters was analyzed using Cramer's V. Classification and regression tree (CRT) analysis was used to identify the cut-offs of variables that best discriminated among clusters. Analyses were carried out using IBM SPSS Statistics, version 20 and Stata, version 13.

\section{Results}

Of the 921 patients participating in the study, 809 completed all SC assessments. Completers were compared with non-completers using t-test and $X^{2}$-test. No difference was found between the two groups on the 29 variables examined except for SLOF interpersonal relationships, that was about 2 points lower among those who did not complete the SC assessment (20.6 vs. 22.5 , t-test=-2.99, $p<0.01)$. Therefore, completers can be considered representative of the overall sample.

Clinical characteristics of completers are provided in Table 1.Concerning milestone achievement, being ever married/engaged was significantly associated with living independently $(\varphi=0.249, p<0.001)$ and with being employed $(\varphi=0.102, p=0.005)$. Although correlations were weak in absolute value, according to Cohen's definition (Cohen, 1988), they were in the expected direction. Specifically, being ever married was more strongly associated with living independently than with being employed, as expected.

Similarly, being employed and living independently were related to each other $(\varphi=0.126, p<0.001)$. Achievement of 1,2 and 3 milestones was found in $38.1 \%, 26.3 \%, 9.4 \%$ of patients; $26.3 \%$ did not achieve any milestone. 


\section{Cluster selection}

The cluster analysis of the $9 \mathrm{SC}$ variables based on the likelihood distance yielded 3 clusters including 340 (42\%), $408(50 \%), 61$ (8\%) patients. The three-cluster solution, depicted in Fig. 1 in a biplot graph, proved to be the best in terms of goodness of fit, yielding a lower value ( $B I C=7283)$ as compared to the 2-and 1-clusters solutions ( $B I C=7654$ and $B I C=8707$ ). An identical solution was found when the Euclidean distance was used. The three clusters were labeled as unimpaired, impaired, and very impaired SC. TASIT LI (lie), SS (simple sarcasm), SA (sarcasm enriched) were the most important variables contributing to the cluster definition. The cluster profile on the 9 standardized variables is depicted in Fig.2, with respect to the reference line of 0 in the control group. This graph shows that mean TASIT and FEIT scales provide a better discrimination among clusters than MSCEIT scores, that tend to be similar in the intermediate and low SC cluster. Moreover, patients in the unimpaired SC cluster exhibited SC impairment in all dimensions investigated, except for TASIT-SI scores, that did not differ significantly between patients and the normative sample.

\section{Correlates of cluster membership}

We found that deficits in SC were associated with older age and lower education, but not with age at onset of psychosis or with gender, alcohol or substance abuse and suicide attempts (Table 2).

Comparison of SLOF functioning scale scores among the 3 clusters using ANOVA revealed that functioning declined significantly from the unimpaired to the impaired and very impaired SC cluster, suggesting a linear correlation between SC and functioning (Table 2). Work and community activities domains were the functioning domains more strongly associated with SC. Moreover, we found that SC had a stronger and positive association with vocational (ever employed, $V=0.142, p<0.001)$ than with social milestones $(V=0.090$, $\mathrm{p}=0.042)$ and was unrelated with living independently $(\mathrm{V}=0.061, \mathrm{p}=0.230)$.

The PANSS disorganization score increased significantly from the unimpaired to the impaired and very impaired SC cluster. The PANSS positive symptoms domain score was significantly lower in unimpaired SC cluster as compared to impaired and very impaired ones, with no differences between them, whereas both BNSS A and DE scores were significantly higher in patients with impaired SC compared to patients in unimpaired SC cluster, with no differences with very impaired SC cluster (Table 3).

Comparison of NC scales scores among clusters showed that NC and SC impairment were strongly related (higher NC impairment, poorer SC) in all the dimensions investigated, except for visuospatial memory (BVMTR) scores, that did not differ significantly between patients with very impaired and impaired SC (Table 3).

\section{Classification and regression tree analysis}


Although the clusters were defined using 9 scales, the scales were highly correlated with each other; therefore we tested the hypothesis that a smaller number of variables would be sufficient to characterize the clusters. To identify the minimum set of variables and the cut-offs for defining the three clusters, we used CRT.

This analysis revealed that two TASIT variables (SS and LI) were sufficient to discriminate among the three clusters. Patients with a cut-off scores $\geq 14$ for TASIT-SS were more likely to have unimnpaired SC, those with a TASIT-SS $<14$ and a TASIT-LI $\geq 10$ were more likely to have impaired SC and those with a TASIT-SS $<14$ and a TASIT-LI<10 were more likely to have very impaired SC (Fig. 3).

\section{Discussion}

To the best of our knowledge, this is the largest study carried out to subtype patients with schizophrenia on the basis of SC.

This study shows several key results relevant to clinical practice.

Firstly, using cross-sectional data, our analysis identified three distinct clusters, characterized by unimpaired (42\%), impaired (50.4\%), and very impaired (7.5\%) SC. Three TOM domains were more important for the cluster definition as compared to emotion perception and emotional intelligence. Moreover, we found specific cut-offs based on levels of impairment on SC measures that can be used in clinical practice.

Second, the three clusters were associated with distinct patterns of real-life functioning, socio-demographic, clinical, and NC variables.

\section{$\mathrm{SC}$ clusters}

In our study two main findings emerged regarding the SC cluster membership.

First, although the three clusters showed SC performance deficits across all measures when compared to healthy controls, except for TASIT-SI,TOM (TASIT LI, SS, SA) was more important for the cluster definition as compared to emotion perception and emotional intelligence. TOM involves the ability to ascertain the mental states of others, and accordingly is likely to affect functioning behaviors to a great extent (Biederman et al, 2012; Couture et al., 2006; Couture et al., 2011).

Second, using the CRT analysis we identified two TASIT scales (SS and LI) and their cut-off values that may provide a useful guidance to clinicians about the patients' degree of impairment in SC. Patients more able to understand simple sarcasm ( $\geq 14$ for TASIT-SS) are very likely to belong to the unimpaired SC cluster. Compared with patients in the impaired SC cluster, those in the very impaired SC cluster performed significantly worse in lie scenes (TASIT-Ll/10), but not in simple sarcasm. Thus, our findings suggest that patients in the unimpaired cluster differ from patients in the impaired and in the very impaired ones in a higher ability to grasp sarcasm, while patients in the impaired cluster differ from those in the very impaired cluster as regards their higher ability to understand lies. This result was not surprising given the findings of studies 
showing that comprehension of lies/deceit is acquired before sarcasm/irony and is based on a less complex inferential chain, reflecting first order mental representation, whereas comprehension of sarcasm requires refined emotional skills such as empathic appreciation of the listener's emotional state (Biederman et al, 2012; Mancuso et al., 2011), reflecting second order mental representation and hierarchically higher-level SC ability. This distinction between lies and sarcasm may have been enhanced by the way that the TASIT evaluates detection of lies as it provides all of the information about deceit in the scene and does not require much inference.

\section{SC and functioning}

Functioning was highest in the unimpaired cluster and deteriorated from the unimpaired to the impaired and very impaired cluster, suggesting a linear correlation between SC and functioning. Work and activities domains were the functioning domains more strongly associated with SC. Our result was confirmed by the findings that patients with high levels of SC were more likely to achieve vocational and social milestones compared with those with lower levels of SC. Moreover, our findings are in line with those of previous meta-analyses, showing that the correlation between SC and real-life functioning ranged from small to large, mainly depending on the examined aspect of SC, with largest effects observed for TOM (Couture et al., 2006, 2011; Fett et al., 2011). The studies reviewed by the Fett meta-analyses evaluated four domains of outcome: community functioning (reported in 33 studies), social behavior in the mileau (reported in 9 studies), social problem solving (reported in 7 studies), and social skills (reported in 9 studies). As regards SC domains, 5 studies investigated TOM (reported in 5 studies), 14 investigated emotion perception and processing (EP), and 8 studies social perception and knowledge (SP). However, the various SC-outcome associations differed in strength. The largest mean correlation was found for the relation between TOM and community functioning, followed by the association between EP and social behavior and the correlation between SP and social skills. No metaanalyses could be performed on social-problem solving and any SC domain due to lack of data. Moreover, it should be noted that in the present study we measured different SC constructs (i.e., emotional intelligence, emotional recognition and TOM) and everyday functioning (ratings of real-life functioning and the achievement of specific milestones) with state of the art assessments

The findings of a previous study (Galderisi et al., 2014) confirm that SC accounts for a unique proportion of functioning variance, independent of NC. Moreover, SC appears to act as a mediator between nonsocial basic NC cognition and community functioning (Schmidt et al, 2011; Galderisi et al, 2014). Lastly, it has been suggested that TOM difficulties play a major role in work functioning as they are important in forming and maintaining social relationships, in achieving social support and personal resources leading to interpersonal difficulties at work. These disturbances may lead to social misperceptions that influence how an individual 
reacts to others, which in turn may lead to maladaptive social patterns and/or social withdrawal, which both may influence real-life vocational outcome more than NC abilities (Couture et al., 2006; Fett et al., 2011).

\section{SC and NC}

A gradient of deterioration across SC clusters was also found for the NC functions, except for visual learning, that was highest in the unimpaired cluster, and did not differ between the impaired and very impaired SC clusters. The relationship between SC and NC functioning is somewhat unclear. A crucial issue that the field is currently facing is about how closely SC processes overlap with NC (Mehta et al., 2013). Despite the fact that basic NC abilities may underlie rapid interpretation of complex social stimuli to inform the moment-to-moment generation, refinement and selection of models for thoughts and emotions of others, which underlie diverse SC abilities (Mehta et al., 2014b), there is some evidence suggesting that a certain level of NC function may be necessary though not sufficient for good SC (Fanning et al., 2012; Hoe et al., 2012). Indeed, conceptually SC involves the interface of socio-emotional and cognitive processing, whereas $\mathrm{NC}$ is considered to be affectneutral (Adolphs, 2009). A recent meta-analysis has shown small to medium range non-specific correlations among different dimensions of these two constructs (Ventura et al., 2013).

Notably, we found that patients in the unimpaired SC cluster performed better in visuospatial memory as compared with patients in the impaired and very impaired SC clusters. Visual working memory tasks involve keeping a visuospatial stimulus, while working on a related or unrelated task. Thus it is a cognitive process which is the foundation of a variety of high level functions like thinking, language and planned behaviors. Accordingly, these findings indicate that individuals in the unimpaired SC cluster could have lower difficulty with higher-level control of visual memory processes, such as use of strategies to enhance encoding and retrieval of novel visual information. Indeed, a visual memory deficit may have several potential negative effects on a range of instrumental activities of daily living from the most common, such as watching TV or reading a book, to the most complex, including social interactions (visual recognition of social signals), recognition of territorial boundaries (interpersonal space) (Cummings and Mega, 2003), autonomy in daily living, and treatment compliance (Prouteau et al., 2005).

Early visual processing was found to have a significant association with emotion recognition and social perception (Kee et al., 1998; Sergi et al., 2006) in schizophrenia. Some authors reported that social perception mediated the relationship between visual perception and functioning (Sergi et al., 2006; Rassovsky et al., 2011). Other studies found that an early visual process (contour integration) is related to the higher-level SC construct of TOM (Schenkel et al., 2005; Uhlhaas et al., 2006). This supports the theoretical connection between perceptual processes and SC based on a cascade model, in which poor perceptual information contributes to inaccurate higher-level information (Javitt, 2009). 


\section{SC and psychopathology}

SC deficits have been previously suggested to be a crucial component in the development of schizophrenia symptoms (Brekke et al., 2005; Couture et al., 2006; Sergi et al., 2007; Addington et al., 2010). A metaanalysis of 154 studies (Ventura et al., 2013) has shown that fluctuations in SC impairments seem to be poorly related to symptoms of reality distortion, but have fairly strong relationships with disorganization and negative symptoms.

Research on the relationship between positive symptoms and SC is complicated by the fact that many studies combine reality distortion and disorganization in their definition of positive symptoms, obscuring which positive symptom is most relevant to which SC process and by the sensitivity of the TOM measurement used.

As regards negative symptoms, several articles have highlighted their role in understanding various SC domains (Mazza et al., 2007; Sergi et al. 2007; Green et al. 2008, 2010). One possibility is that negative symptoms that involve reduced emotional experience (ie, anhedonia) or expression (ie, affective flattening) might be more associated with the development or maintenance of SC deficits (Sergi et al., 2007), apparently because of difficulties representing the mental states of others as well as themselves. Negative symptoms are also associated with lower levels of complexity of social representations, poor social adjustment and impaired capacity for emotional investment (Biederman et al., 2012). The current finding that negative symptoms were highest in patients with impaired SC, with no difference between unimpaired SC and very impaired SC cluster may be partly explained by the small size of this latter group and its the large variability.

As for disorganization, the correlation with SC in our study is in line with the literature (Hardy-Bayle' et al., 2003; Abdé-Amil et al., 2009). Symptoms of disorganization have been associated with failure to take into account the intentions of others, as well as impairments in causal attributions, accuracy of character ascriptions, and integration of social episodes. Moreover, patients with disorganized schizophrenia have difficulty in using contextual information to select an appropriate response in a way that is appropriate to the situation. On the other hand, it has been suggested that a difficulty in understanding other people's mental states, i.e., a TOM deficit, could induce signs such as poor, incoherent, or inappropriate speech, i.e. disorganized symptoms. Of all the situations encountered in everyday life, communication with others requires the greatest ability to adapt to the context and to attribute mental states: a conversation, consisting as it does of verbal and nonverbal exchanges with other people, is by definition a shifting and uncertain context, and the data associated with it must be constantly inferred and updated on the basis of peripheral information (Leslie, 1987).Taken together, our results provide support for the notion that positive symptoms and disorganization represent separate dimensions with differential links to SC in schizophrenia.

\section{Strengths and limitations}


The study had some limitations that should be highlighted. First, because of the cross-sectional design, we were not able to determine the natural stability of these SC subtypes over the course of illness. Second, to be eligible for this study patients had to be outpatients meeting criteria for psychiatric stability, consequently they were not representative of patients in acute phases or in other clinical settings. Third, it should be noted that cluster analysis is exploratory in nature and the results are highly dependent on the method used to aggregate individuals by similarity measures. Still, the clusters proved to be robust and to be associated with specific sociodemographic, psychopathology and functioning profiles.

Despite these limitations, there are some strengths: the large sample size, the use of state-of-the-art instruments to assess real-life functioning, NC, psychopathological, SC variables, and the naturalistic design without selection bias related to randomized controlled designs. Indeed, since data from randomized controlled trials provide efficacy data in a relatively homogenous population under artificial circumstances, it is reassuring to find that these results are confirmed in usual practice real-life settings. Indeed, as underlined by Aldenderfer and colleagues (1984), if a cluster solution is repeatedly discovered across different samples from the same general population, it is reasonable to conclude that it has some kind of general utility.

\section{Conclusions}

In conclusion, we identified three distinct clusters of SC performance in patients with schizophrenia. Moreover, we identified a parsimonious subset of TOM (TASIT SS and TASIT LI) and their specific cut-off scores that can be used in clinical practice to discriminate patients with different levels of SC impairment. 


\section{References}

Abdel-Hamid M, Lehmka"mper C, Sonntag C, Juckel G, Daum I, Brüne M (2009). Theory of mind in schizophrenia: the role of clinical symptomatology and neurocognition in understanding other people's thoughts and intentions. Psychiatry Research 165, 19-26.

Abu-Akel A, Bailey AL (2000). The possibility of different forms of theory of mind impairment in psychiatric and developmental disorders. Psychological Medicine 30, 735-738.

Addington J, Girard TA, Christensen BK, Addington D (2010). Social cognition mediates illness-related and cognitive influences on social function in patients with schizophrenia-spectrum disorders. Journal of Psychiatry \& Neuroscience 35, 49-54.

Adolphs R (2009). The social brain: neural basis of social knowledge. Annual Review of Psychology 60, 693716.

Aldenderfer MS, Blashfield RK (1984). Cluster analysis. Sage Publications: Beverly Hills, CA.

Bell M, Tsang HWH, Greig TC, Bryson GJ (2009). Neurocognition, social cognition, perceived social discomfort, and vocational outcomes in schizophrenia. Schizophrenia Bulletin 35, 738-747.

Bell MD, Corbera S, Johannesen JK, Fiszdon JM, Wexler BE (2013). Social cognition impairment and negative symptoms in schizophrenia; are there subtypes with distinct functional correlates? Schizophrenia Bulletin 39, 186-196.

Biedermann F, Frajo-Apor B, Hofer A (2012). Theory of mind and its relevance in schizophrenia. Current Opinion in Psychiatry 25, 71-5.

Bora E, Pantelis C (2013). Theory of mind impairments in first-episode psychosis, individuals at ultra-high risk for psychosis and in first-degree relatives of schizophrenia: systematic review and meta-analysis. Schizophrenia Research 144, 31-6.

Brekke J, Kay DD, Lee KS, Green MF (2005). Biosocial pathways to functional outcome in schizophrenia. Schizophrenia Research 80, 213-225.

Brown EC, Tas C, Cand H, Esen-Danacie A, Brüne M (2014). A closer look at the relationship between the subdomains of social functioning, social cognition and symptomatology in clinically stable patients with schizophrenia. Comprehensive Psychiatry 55, 25-32.

Chung YS, Mathews JR, Barch DM (2010). The effect of context processing on different aspects of social cognition in schizophrenia. Schizophrenia Bulletin 37, 1048-1056.

Cohen J (1988) Statistical Power Analysis for the Behavioral Sciences, 2nd ed., Erlbaum, Hillsdale, NJ.

Couture SM, Penn DL, Roberts DL (2006). The functional significance of social cognition in schizophrenia: a review. Schizophrenia Bulletin 32 Suppl.1, S44-S63. 
Couture SM, Granholm EL, Fish SC (2011). A path model investigation of neurocognition, theory of mind, social competence, negative symptoms and real-world functioning in schizophrenia. Schizophrenia Research $125,152-160$.

de Achával D, Costanzo EY, Villarreal M, Jáuregui IO, Chiodi A, Castro MN, Fahrer RD, Leiguarda RC, Chu EM, Guinjoan SM (2010). Emotion processing and theory of mind in schizophrenia patients and their unaffected first-degree relatives. Neuropsychologia 48, 1209-1215.

Cummings JL, Mega MS (2003)."Visuospatial, visuoperceptual, and right hemisphere disturbances" in Neuropsychiatry and Behavioral Neuroscience, eds Cummings JL and Mega MS (NewYork, NY: Oxford University Press), 114-127.

Fanning JR, Bell MD, Fiszdon JM (2012). Is it possible to have impaired neurocognition but good social cognition in schizophrenia? Schizophrenia Research 135, 68-71.

Fett AKJ, Viechtbauer W, Dominguez MG, Penn DL, van Os J, Krabbendam L (2011). The relationship between neurocognition and social cognition with functional outcomes in schizophrenia: a meta-analysis. Neuroscience \&Biobehavioral Reviews 35, 573-588.

Fett AK, Maat A; GROUP Investigators (2013). Social cognitive impairments and psychotic symptoms: what is the nature of their association? Schizophrenia Bulletin 39, 77-85.

Fiszdon J, Fanning JR, .Johannesen JK, Bell MD (2013). Social cognitive deficits in schizophrenia and their relationship to clinical and functional status. PsychiatryResearch 205, 25-29.

Galderisi S, Rossi A, Rocca P, Bertolino A, Mucci A, Bucci P, Rucci P, Gibertoni D, Aguglia E, more M, Bellomo A, Biondi M, Brugnoli R, Dell'Osso L, De Ronchi D, Di Emidio G, Di Giannantonio M, Fagiolini A, Marchesi C, Monteleone P, Oldani L, Pinna F, Roncone R, Sacchetti E, Santonastaso P, Vita A, Zeppegno P, Maj M; Italian Network For Research On Psychoses (2014).The influence of illness-related variables, personal resources and context-related factors on real-life functioning of people with schizophrenia. World Psychiatry 13, 275-287.

Green MF, Bearden CE, Cannon TD, Fiske AP, Hellemann GS, Horan WP, Kee K, Kern RS, Lee J, Sergi MJ, Subotnik KL, Sugar CA, Ventura J, Yee CM, Nuechterlein KH (2012). Social cognition in schizophrenia, part 1: performance across phase of illness. Schizophrenia Bulletin 38, 854-864.

Green MF, Horan WP (2010). Social cognition in schizophrenia. Current Directions in Psychological Science 19, 243-248.

Green MF, Penn DL, Bentall R, Carpenter WT, Gaebel W, Gur RC, Kring AM, Park S, Silverstein SM, Heinssen R (2008). Social cognition in schizophrenia: an NIMH workshop on definitions, assessment, and research opportunities. Schizophrenia Bulletin 34, 1211-1220.

Hardy-Bayle' MC, Sarfati Y, Passerieux C (2003). The cognitive basis of disorganization symptomatology in schizophrenia and its clinical correlates. Schizophrenia Bulletin 29, 459-471. 
Harvey PD (2013). Assessment of everyday functioning in schizophrenia: implications for treatments aimed at negative symptoms. Schizophrenia Research 150, 353-5.

Harvey PD, Penn DL (2010). Social cognition: The key factor predicting social outcome in people with schizophrenia? Psychiatry 7, 41-44.

Harvey PD, Raykov T, Twamley EW, Vella L, Heaton RK, Patterson TL (2011). Validating the measurement of real-world functional outcomes: phase I results of the VALERO study. The American Journal of Psychiatry $168,1195-1201$.

Harvey PD, Sabbag S, Prestia D, Durand D, Twamley EW, Patterson TL (2012). Functional milestones and clinician ratings of everyday functioning in people with schizophrenia: overlap between milestones and specificity of ratings. Journal of Psychiatric Research 46, 1546-52.

Hoe M, Nakagami E, Green MF, Brekke JS (2012).The causal relationships between neurocognition, social cognition and functional outcome over time in schizophrenia: a latent difference score approach. Psychological Medicine 42, 2287-2299.

Horton HK, Silverstein SM (2008). Social cognition as a mediator of cognition and outcome among deaf and hearing people with schizophrenia. Schizophrenia Research 105, 125-137.

Javitt DC (2009). When doors of perception close: bottom-up models of disrupted cognition in schizophrenia. Annual Review of Clinical Psychology 5, 249-275.

Kee KS, Kern RS, Green MF (1998). Perception of emotion and neurocognitive functioning in schizophrenia: what's the link? Psychiatry Research 81, 57-65.

Kern RS, Nuechterlein KH, Green MF, Baade LE, Fenton WS, Gold JM, Keefe RS, Mesholam-Gately R, Mintz J, Seidman LJ, Stover E, Marder SR (2008). The MATRICS Consensus Cognitive Battery, part 2: conorming and standardization. The American Journal of Psychiatry 165, 214-220.

Kerr SL, Neale JM (1993). Emotion perception in schizophrenia: specific deficit or further evidence of generalized poor performance? Journal of Abnormal Psychology 102, 312-318.

Kirkpatrick B, Strauss GP, Nguyen L, Fischer BA, Daniel DG, Cienfuegos A, Marder SR (2011). The Brief Negative Symptom Scale: psychometric properties. Schizophrenia Bulletin 37, 300-305.

Kohler CG, Walker JB, Martin EA, Healey KM, Moberg PJ (2010). Facial emotion perception in schizophrenia: a meta-analytic review. Schizophrenia Bulletin 36, 1009-1019.

Leifker FR, Patterson TL, Heaton RK, Harvey PD (2011). Validating measures of real-world outcome: the results of the VALERO expert survey and RAND panel. Schizophrenia Bulletin 37, 334-343.

Leslie AM, Frith U (1987). Metarepresentation and autism: how not to lose one's marbles. Cognition 27, 291294.

Mancuso F, Horan WP, Kern RS, Green MF (2011). Social cognition in psychosis: Multidimensional structure, clinical correlates and relationship with functional outcome. Schizophrenia Research 125, 143-151. 
Martínez-Domínguez S, Penadés R, Segura B, González-Rodríguez, Catalán R (2015). Influence of social cognitionon daily functioning in schizophrenia: Study of incremental validity and mediational effects. Psychiatry Research 225, 374-380.

Mayer JD, Salovey P, Caruso DR. (2002).Mayer-Salovey-Caruso Emotional Intelligence Test (MSCEIT): Users' Manual. Multi-Health Systems Inc: Toronto.

Mazza M, Costagliola C, Di Michele V, Magliani V, Pollice R, Ricci A, Di Giovanbattista E, Roncone R, Casacchia M, Galzio RJ (2007). Deficit of social cognition in subjects with surgically treated frontal lobe lesions and in subjects affected by schizophrenia. European Archives of Psychiatry and Clinical Neuroscience 257, 12-22.

McDonald S, Bornhofen C, Shum D, Long E, Saunders C, Neulinger K. (2006). Reliability and validity of The Awareness of Social Inference Test (TASIT): a clinical test of social perception. Disability and Rehabilitation 28, 1529-1542.

Mehta UM, Thirthalli J,Subbakrishna DK., Gangadhar BN, Eack SM, Keshavan MS (2013).Social and neuro-cognition as distinct cognitive factors in schizophrenia: a systematic review. Schizophrenia Research $148,3-11$.

Mehta UM, Bhagyavathi HD, Thirthalli J,. Kumar KJ, Gangadhar BN (2014). Neurocognitive predictors of social cognition in remitted schizophrenia. Psychiatry Research 219, 268-274.

Mucci A, Galderisi S, Merlotti E, Rossi A, Rocca P, Bucci P, Piegari G, Chieffi M, Vignapiano A, Maj M (2015) Italian Network for Research on Psychoses. The Brief Negative Symptom Scale (BNSS): Independent validation in a large sample of Italian patients with schizophrenia. European Psychiatry 30, 641-647.

Mucci A, Rucci P, Rocca P, Bucci P, Gibertoni D, Merlotti E, Galderisi S, Maj M (2014). Italian Network for Research on Psychoses. The Specific Level of Functioning Scale: construct validity, internal consistency and factor structure in a large sample of people with schizophrenia living in the community. Schizophrenia Research 159, 144-150.

Nelson AL, Combs DR, Penn DL, Basso MR(2007). Subtypes of social perception deficits in schizophrenia. Schizophrenia Research 94, 139-147.

Norušis MJ (2005). IBM SPSS Statistics 19 Statistical Procedures Companion, Prentice Hall, NJ. Nuechterlein KH, Green MF, Kern RS, Baade LE, Barch DM, Cohen JD, Essock S, Fenton WS, Frese FJ 3rd, Gold JM, Goldberg T, Heaton RK, Keefe RS, Kraemer H, Mesholam-Gately R, Seidman LJ, Stover E, Weinberger DR, Young AS, Zalcman S, Marder SR (2008). The MATRICS Consensus Cognitive Battery, part 1: test selection, reliability, and validity. The American Journal of Psychiatry 165, 203-213.

Nuechterlein KH, Green MF (2013a). MATRICS Consensus Cognitive Battery (MCCB): history and development of the battery - Translation of chapters 1 and 2 of the MCCB Manual by F Mancuso, A Mucci, $S$ Galderisi. NOOS 2, 83-98. 
Nuechterlein KH, Green MF (2013b). MATRICS Consensus Cognitive Battery (MCCB): reliability, validity and standardization studies - Translation of chapters 1 and 2 of the MCCB Manual by F Mancuso, A Mucci, S Galderisi. NOOS 2, 99-116.

Pinkham AE, Penn DL, Green MF, Harvey PD (2015). Social Cognition Psychometric Evaluation: Results of the Initial Psychometric Study. Schizophrenia Bulletin 4.

Prouteau A, Verdoux H, Briand C, Lesage A, Lalonde P, Nicole L, Reinharz D, Stip E (2005).Cognitive predictors of psychosocial functioning outcome in schizophrenia: a follow-up study of subjects participating in a rehabilitation program. Schizophrenia Research 77, 343-353.

Rassovsky Y, Horan WP, Lee J, Sergi MJ, Green MF (2011). Pathways between early visual processing and functional outcome in schizophrenia. Psychological Medicine 41, 487-497.

Savla GN, Vella L, Armstrong CC, Penn DL, Twamley EW (2013). Deficits in domains of social cognition in schizophrenia: a meta-analysis of the empirical evidence. Schizophrenia Bulletin 39, 979-992.

Schenkel LS, Spaulding WD, Silverstein SM (2005). Poor premorbid social functioning and theory of mind deficit in schizophrenia: evidence of reduced context processing? Journal of Psychiatric Research 39, 499508.

Schmidt SJ, Mueller DR, Roder V (2011). Social cognition as a mediator variable between neurocognition and functional outcome in schizophrenia: empirical review and new results by structural equation modeling. Schizophrenia Bulletin 37 Suppl.2, S41-S54.

Schneider LC, Struening EL (1983). SLOF: a behavioral rating scale for assessing the mentally ill. Social work research \& abstracts 19, 9-21.

Sergi MJ, Rassovsky Y, Nuechterlein KH, Green MF (2006). Social perception as a mediator of the influence of early visual processing on functional status in schizophrenia. The American Journal of Psychiatry $163,448-454$.

Sergi MJ, Rassovsky Y, Widmark C, Reist C, Erhart S, Braff DL, Marder SR, Green MF (2007). Social cognition in schizophrenia: relationships with neuro-cognition and negative symptoms. Schizophrenia Research 90, 316-324.

Strauss GP, Keller WR, Buchanan RW, Gold JM, Fischer BA, McMahon RP, Catalano LT, Culbreth AJ, Carpenter WT, Kirkpatrick B (2012). Next-generation negative symptom assessment for clinical trials: validation of the Brief Negative Symptom Scale. Schizophrenia Research 142, 88-92.

Ventura J, Wood RC, Hellemann GS (2013). Symptom Domains and Neurocognitive Functioning Can Help Differentiate Social Cognitive Processes in Schizophrenia: A Meta-Analysis. Schizophrenia Bulletin 39, 102111. 
Wallwork RS, Fortgang R, Hashimoto R, Weinberger DR, Dickinson D (2012). Searching for a consensus five-factor model of the Positive and Negative Syndrome Scale for schizophrenia. Schizophrenia Research 137, 246-250. 